Annales Academiæ Scientiarum Fennicæ

Mathematica

Volumen 42, 2017, 803-808

\title{
MEASURABILITY OF SIMILAR FUNCTIONS
}

\author{
Ivan Kupka \\ Comenius University, Faculty of Mathematics, Physics and Informatics \\ Department of Mathematical Analysis and Numerical Mathematics \\ Mlynska Dolina, Bratislava, Slovakia; ivan.kupka@seznam.cz
}

\begin{abstract}
We present new sufficient conditions for functions to be measurable, or Darboux, or to be of the first Baire class. Two main results are obtained in a general topological context.
\end{abstract}

\section{Introduction}

In this article we give some new sufficient conditions for functions to be measurable. We also show that similarly formulated conditions are sufficient for functions to be of the first Baire class $\left(B_{1}\right)$ or to belong to the classes $B_{1}^{*}, B_{1}^{* *}[13]$ or to be Darboux.

So-called relations of continuity and constancy were defined in [7]. They enable to compare functions with unknown properties with "nice" functions. This approach allows also to generalize some continuity-preserving theorems and to generate new ones. Sometimes it allows to replace differentiation by manipulation with inequalities - so it can be used to examine nondifferentiable functions. Optimization applications of this approach have been shown in [8].

\section{Basic notions}

In what follows we will use these notions: a net of points, a limit of a net, a net of functions, uniform convergence, pointwise convergence (see e.g. [2] or [3]).

Let $X=\left(X, \sum\right)$ be a measurable space, where $\Sigma$ is a $\sigma$-algebra on $X$, let $Y=$ $(Y, T)$ be a topological space. We say that a function $f: X \rightarrow Y$ is measurable, if for every $G$ from $T$ the set $f^{-1}(G)$ is from $\Sigma$.

Let $X, Y$ be topological spaces. A function $f$ from $X$ to $Y$ is Darboux if and only if it takes every connected set to a connected set. We say that a function $f$ from a metric space $X$ to a metric space $Y$ belongs to the class $B_{1}$, or that it is of the first Baire class, if for every open set $O$ in $Y$ the set $f^{-1}(O)$ is $F_{\sigma}$. The investigation of the class $B_{1}$ started very early, see Baire's article [1].

\section{Dominance and measurability}

In this section we present new sufficient conditions for functions to be measurable. The main result of this section is Theorem 3.6. Now we define a useful notion, the notion of $f$-dominance.

https://doi.org/10.5186/aasfm.2017.4246

2010 Mathematics Subject Classification: Primary 26A21 28A20; Secondary 26A15, 54C10, $54 \mathrm{D} 05$.

Key words: Continuously similar functions, strongly similar functions, measurable functions, first Baire class, Darboux functions. 
Definition 3.1. Let $\left(X, \tau_{1}\right),\left(Y, \tau_{2}\right)$ be topological spaces, $A \subset X,(Z, d)$ and $(V, \varrho)$ be metric spaces. Let $f: X \rightarrow Y, g: X \rightarrow Z$ be functions. If there exists a continuous function $h: Y \rightarrow V$ such that

(dom) $\exists L>0: \forall x_{1}, x_{2} \in A d\left(g\left(x_{1}\right), g\left(x_{2}\right)\right) \leq L \varrho\left(h\left(f\left(x_{1}\right)\right), h\left(f\left(x_{2}\right)\right)\right)$,

we say that $g$ is $f$-dominated on $A$. If $x \in X$ and $g$ is $f$-dominated on an open neighborhood of $x$, we say that $g$ is $f$-dominated at $x$. If $A=X$, we say simply that $g$ is $f$-dominated.

Now we introduce two notions of similarity of functions (defined in [7]). Here we are using the terminology used in [8].

Definition 3.2. (i) Let $X, Y, Z$ be topological spaces, let $f: X \rightarrow Y, g: X \rightarrow Z$ be functions. Let $x \in X$. We say that $g$ is $f$-continuous at $x$ if for every net $\left\{x_{\gamma}\right\}_{\gamma \in \Gamma}$ of elements from $X$ converging to $x$ the following holds:

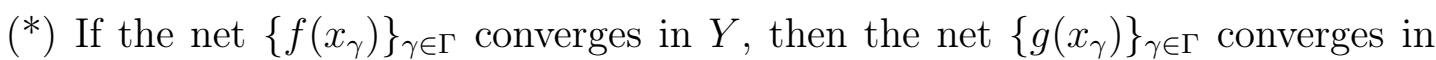
$Z$.

Let $A$ be a subset of $X$. We say that $g$ is $f$-continuous on $A$ if for every $x \in A g$ is $f$-continuous at $x$. If $A=X$, we say that $g$ is $f$-continuous.

(ii) Let $X$ be a set, $Y, Z$ be topological spaces. Let $f: X \rightarrow Y, g: X \rightarrow Z$ be functions. Let $P$ be a nonempty subset of $X$. We say that $g$ is $f$-constant on $P$ if for every net $\left\{x_{\gamma}\right\}_{\gamma \in \Gamma}$ of elements from $P$ the following holds

(**) If the net $\left\{f\left(x_{\gamma}\right)\right\}_{\gamma \in \Gamma}$ converges in $Y$, then the net $\left\{g\left(x_{\gamma}\right)\right\}_{\gamma \in \Gamma}$ converges in $Z$.

If $P=X$, we say simply that $g$ is $f$-constant. (The $f$-continuity of $g$ per se does not guarantee that $g$ will automatically have all nice properties of $f$. It must be always examined, whether a property of $f$ is inherited by $g$ or not.)

Remark 3.3. It is known that a function from a topological space $X$ into a Hausdorff space $Y$ is continous at $x \in X$ if an only if for each net $\left\{x_{\gamma}\right\}_{\gamma \in \Gamma}$ converging to $x$ the net $\left\{f\left(x_{\gamma}\right)\right\}_{\gamma \in \Gamma}$ converges in $Y$ [5]. So we can see immediately that if $Y, Z$ are Hausdorff and $f$ is continuous on a subset $A$ of $X$ and $g$ is $f$-continuous at every $x \in A$, then $g$ is continuous on $A$ too. And if $g$ is not continuous at a point $x \in X$ and it is $f$-continuous at $x$, then $f$ is not continuous at $x$.

If the function $g$ has values in a complete metric space $(Y, d)$ and $f$ has values in a metric space $(Z, \rho)$ the following is obvious:

(i) if there exists a positive constant $K$ such that for all points $t, s$ from an open neighborhood of $x$ (for all points $t$ and $s$ from $X$ )

$$
d(g(t), g(s)) \leq K \cdot \rho(f(t), f(s))
$$

is true, then $g$ is $f$-continuous at $x$ ( $g$ is $f$-constant).

(ii) If $g$ is $f$-dominated at a point $x$, then it is $f$-continuous at $x$. And if $g$ is $f$-dominated (on $X$ ), then it is $f$-constant.

The following example should help the reader to get a first insight into the new notions.

Example 3.4. a) Let $Y=\langle 0,1\rangle, X=Z=(0,1)$. Define $g: X \rightarrow Y$ and $f: X \rightarrow Z$ by

for all $x \in X g(x)=x$ and $f(x)=x$.

Although $g$ and $f$ are very similar (only $Y$ and $Z$ differ a little bit), we can see that $g$ is $f$-constant but $f$ is not $g$-constant-the net $\left\{g\left(\frac{1}{n}\right)\right\}_{n \in \mathbf{N}}$ converges but the net 
$\left\{f\left(\frac{1}{n}\right)\right\}_{n \in \mathbf{N}}$ does not converge. It is easy to check that $f$ is $g$-continuous and $g$ is $f$-continuous.

b) When $X, Y, Z$ are arbitrary topological spaces and $p: X \rightarrow Y$ and $q: X \rightarrow Z$ are functions, if $p$ is continuous at a point $x \in X$ then $p$ is $q$-continuous at the point $x$. And if $p$ is constant on $X$, then $p$ is $q$-constant.

The following lemma shows two ways, in which the notions of $f$-constancy and $f$-continuity are connected. The validity of the lemma follows from the definitions presented above.

Lemma 3.5. Let $X$ be a set, $(Y, S),(Z, \tau)$ be topological spaces. Let $f: X \rightarrow$ $(Y, S), g: X \rightarrow(Z, \tau)$ be functions.

(i) Let $g$ be $f$-constant on $X$. Then $g:(X, \varphi) \rightarrow(Z, \tau)$ is $f$-continuous on $(X, \varphi)$ for any topology $\varphi$ on $X$. (For the sake of simplicity we continue to use the letters $f$ and $g$ although - from a topological point of view — we are dealing with different functions.)

(ii) The function $g$ is $f$-constant on $X$ if and only if $g:(X$, Triv $) \rightarrow(Z, \tau)$ is $f$-continuous with respect to $f:(X$, Triv $) \rightarrow(Y, S)$-where Triv $=\{X, \emptyset\}$ is the trivial topology on $X$.

Now we are ready for the main result of this section. It states that if a function $f$ is measurable and $g$ is $f$-constant, then $g$ is measurable too. As an obvious consequence we obtain that $f$-dominated functions - defined on the same measurable space as $f$ are measurable.

Theorem 3.6. Let $(X, \Sigma)$ be a measurable space, be $(Y, T),(Z, \tau)$ Hausdorff topological spaces. Let $f: X \rightarrow Y, g: X \rightarrow Z$ be functions. Let $g$ be $f$-constant on $X$. Then the following holds:

(*) If $f$ is measurable then $g$ is measurable too.

Proof. Define a new topology $\eta$ in the following way: $\eta=\left\{f^{-1}(V): V \in T\right\}$. Of course, the function $h:(X, \eta) \rightarrow(Y, T)$ defined by

$$
\forall x \in X h(x)=f(x)
$$

is continuous on $(X, \eta)$. Now we will prove that $g$ is continuous on $(X, \eta)$. According to Remark 3.3 it suffices to prove, that $g$ is $h$-continuous on $(X, \eta)$. To prove this, suppose, that $\left\{x_{\gamma}\right\}_{\gamma \in \Gamma}$ is a net in $X$, converging in the topology $\eta$ to a point $x_{0} \in X$ and such that the net $\left\{h\left(x_{\gamma}\right)\right\}_{\gamma \in \Gamma}$ converges in $(Y, T)$. We need to prove that the net $\left\{g\left(x_{\gamma}\right)\right\}_{\gamma \in \Gamma}$ converges in $(Z, \tau)$. But this is trivial. Indeed: Since $\left\{h\left(x_{\gamma}\right)\right\}_{\gamma \in \Gamma}$ converges in $(Y, T),\left\{f\left(x_{\gamma}\right)\right\}_{\gamma \in \Gamma}$ converges in $(Y, T)$ too. Now we use the fact, that $g$ is $f$-constant. This fact implies the net $\left\{g\left(x_{\gamma}\right)\right\}_{\gamma \in \Gamma}$ converges in $(Z, \tau)$. The function $g:(X, \eta) \rightarrow Z$ is proven to be continuous.

Now, to prove the measurability of $g$ it suffices to show, that for every open set $U$ from $Z$ the set $g^{-1}(U)$ is from $\Sigma$. But if we take an arbitrary $U \in \tau$, the set $g^{-1}(U)$ is an element of the topology $\eta$. From the construction of $\eta$ we can see, that $\eta \subseteq \Sigma$. So the set $g^{-1}(U)$ is proven to belong to $\Sigma$. This ends the proof.

\section{The class $B_{1}$, the Darboux property}

While measurability is preserved under a strong condition, the property being of the first Baire class is conserved (in the metric context) under a milder conditionwhen $g$ is $f$-continuous. In our proof, we use this fact: if $X$ and $Y$ are complete 
separable metric spaces, a function $f: X \rightarrow Y$ is $B_{1}$ if and only if the restriction $f_{P}$ of $f$ to any nonempty closed subset $P$ of $X$ has a point of continuity. [9]

Theorem 4.1. Let $X, Y, Z$ be complete separable metric spaces, let $f: X \rightarrow Y$, $g: X \rightarrow Z$ be functions. If $f$ is of the first Baire class and $g$ is $f$-continuous on $X$, then $g$ is of the first Baire class too.

Proof. The proof is straightforward. Be $P$ a nonempty closed subset of $X$. Since $f$ is of the first Baire class, the restriction $f_{P}$ of $f$ to $P$ has a point of continuity $c_{P}$. This means that for every net $\left\{x_{\gamma}\right\}_{\gamma \in \Gamma}$ of elements from $P$ converging to $c_{P}$ the net $\left\{f\left(x_{\gamma}\right)\right\}_{\gamma \in \Gamma}=\left\{f_{P}\left(x_{\gamma}\right)\right\}_{\gamma \in \Gamma}$ converges to $f\left(c_{P}\right)$. Since $g$ is $f$-continuous, the net $\left\{g_{P}\left(x_{\gamma}\right)\right\}_{\gamma \in \Gamma}=\left\{g\left(x_{\gamma}\right)\right\}_{\gamma \in \Gamma}$ converges too, concretely to the point $g\left(c_{P}\right)$ (we are applying Remark 3.3 to $f_{P}$ and $g_{P}$ ). Therefore $g_{P}$ is continuous at $c_{P}$. So the sufficient condition for $g$ being of the first Baire class is satisfied.

Similar results can be obtained for functions from the classes $B_{1}^{*}, B_{1}^{* *}[11,13]$. We say that a function $f: \mathbf{R} \rightarrow \mathbf{R}$ belongs to the class $B_{1}^{*}$ if for every closed set $C$ there is an open interval $I=(a, b)$ with $I \cap C \neq \emptyset$ such that the restriction $f_{C}$ is continuous on $I$ [11]. We say that a function $f: X \rightarrow Y$ (where $X$ and $Y$ are topological spaces) belongs to the class $B_{1}^{* *}$ if either $D(f)=\emptyset$ or $f_{D(f)}$ is a continuous function-where $D(f)$ denotes the set of all discontinuity points of $f[13]$.

Using the same argument as in the proof of the preceding theorem, we can realize, that the following is true:

Lemma 4.2. Let $X$ be a topological space, $Y, Z$ be Hausdorff topological spaces. Let $f: X \rightarrow Y$ belong to the class $B_{1}^{* *}$ (let $f: \mathbf{R} \rightarrow \mathbf{R}$ belong to the class $B_{1}^{*}$ ) and let $g: X \rightarrow Z(g: \mathbf{R} \rightarrow \mathbf{R})$ be $f$-continuous on $X$. Then $g$ belongs to the class $B_{1}^{* *}$ (then $g$ belongs to the class $B_{1}^{*}$ ) too.

Theorem 4.1 says also this: The property of being of the class $B_{1}$ is inherited from a function $f$ by all $f$-dominated functions (dominated at each point $x$ from the common domain $X$ ).

The family of Darboux functions contains many important classes of mappings, for example derivatives and approximately continuous functions. In what follows we will give a sufficient condition for a function to be Darboux and the condition will be formulated in a purely topological way. In the following theorem we need the dominance on the whole $X$ to assure the inheritance of the Darboux property.

Let us note, that a subset $C$ of a topological space $X$ is connected if and only if for every pair of disjoint sets $X_{1}, X_{2} \subset X$ such that $C=X_{1} \cup X_{2}$ one of the sets $\overline{X_{1}} \cap X_{2}$ or $X_{1} \cap \overline{X_{2}}$ is nonempty. (See e.g. [3])

Theorem 4.3. Let $X \neq \emptyset$ be a set, be $(Y, S),(Z, \tau)$ Hausdorff topological spaces. Let $f: X \rightarrow Y, g: X \rightarrow Z$ be functions. Let $P \subset X$ be nonempty and such that the set $f(P)$ is connected. Let $g$ be $f$-constant on $P$. Then the set $g(P)$ is connected too.

Proof. To prove that the set $g(P)$ is connected in $Z$ it suffices to prove, that for any two nonempty sets $A$ and $B$ such that $g(P)=A \cup B$ the following is true:

(*) $\bar{A} \cap B \neq \emptyset$ or $A \cap \bar{B} \neq \emptyset$.

Let us denote $G_{A}=g^{-1}(A) \cap P$ and $G_{B}=g^{-1}(B) \cap P$. Of course, $P=G_{A} \cup G_{B}$. Now let us denote $U_{A}=f\left(G_{A}\right), U_{B}=f\left(G_{B}\right)$ and $U=f(P)$. The set $U$ is connected, therefore one of the sets $\overline{U_{A}} \cap U_{B}$ or $U_{A} \cap \overline{U_{B}}$ is nonempty. Without loss of generality 
we can suppose that $\overline{U_{A}} \cap U_{B} \neq \emptyset$. From this we have that there exists a net $\left\{y_{\lambda}\right\}_{\lambda \in \Lambda}$ of points from $U_{A}$ converging to a point $b \in U_{B}$.

Let us define a new directed set $\Lambda$ ' by

$$
\Lambda^{\prime}=\{[\lambda, 1] ; \lambda \in \Lambda\} \cup\{[\lambda, 2] ; \lambda \in \Lambda\}
$$

and $\Lambda$ ' be equipped with a preorder defined by

$$
\forall \alpha, \beta \in \Lambda \text { if } \alpha<\beta \text {, then }[\alpha, 1]<[\alpha, 2]<[\beta, 1]<[\beta, 2] .
$$

It is easy to check that $\Lambda^{\prime}$ is a directed set. Next by the symbol $\left\{y_{\lambda}, b\right\}$ we denote the following net: $\left\{y_{\lambda}, b\right\}=\left\{y_{\lambda^{\prime}}\right\}_{\lambda^{\prime} \in \Lambda^{\prime}}$ where for all $\lambda \in \Lambda$ we have $y_{[\lambda, 1]}=y_{\lambda}, y_{[\lambda, 2]}=b$. We can see immediately that the net $\left\{y_{\lambda}\right\}_{\lambda \in \Lambda}$ is a subnet of $\left\{y_{\lambda}, b\right\}$ and that the constant net $\left\{y_{[\lambda, 2]}\right\}_{\lambda \in \Lambda}$ is a subnet of $\left\{y_{\lambda}, b\right\}=\left\{y_{\lambda^{\prime}}\right\}_{\lambda^{\prime} \in \Lambda^{\prime}}$ too.

Let us observe that the "alternate" net $\left\{y_{\lambda}, b\right\}_{\lambda \in \Lambda}$ converges to $b$ too. Because of the definition of $U_{A}$ and $U_{B}$ there exists a point $s \in G_{B}$ such that $f(s)=b$ and there exists a net $\left\{x_{\lambda}\right\}_{\lambda \in \Lambda}$ of points from $G_{A}$ such that for every $\lambda \in \Lambda f\left(x_{\lambda}\right)=y_{\lambda}$. Of course, the alternate net $\left\{f\left(x_{\lambda}\right), f(s)\right\}_{\lambda \in \Lambda}$ (which is equal to the net $\left\{y_{\lambda}, b\right\}_{\lambda \in \Lambda}$ ) is convergent, it converges to the point $b$. Since $g$ is $f$-constant on $P$, the net $\nu=\left\{g\left(x_{\lambda}\right), g(s)\right\}_{\lambda \in \Lambda}$ (defined by the the same procedure as the net $\left\{y_{\lambda}, b\right\}_{\lambda \in \Lambda}$ was) converges too. It converges to the point $g(s) \in B$. The net $\left\{g\left(x_{\lambda}\right)\right\}_{\lambda \in \Lambda}$ is a subnet of $\nu$ so it converges to $g(s)$ too. This means that $\bar{A} \cap B \neq \emptyset$. This ends the proof.

Corollary 4.4. Let $(X, T)$ be a topological space, be $(Y, S),(Z, \tau)$ Hausdorff topological spaces. Let $f: X \rightarrow Y, g: X \rightarrow Z$ be functions. Let $f$ be a Darboux function. If $g$ is $f$-constant on $X$ then $g$ is a Darboux function.

The following example illustrates, that some assumptions of our theorem are essential.

Example 4.5. a) Let $X=\langle 0,1\rangle$ with the usual topology and $Y=Z=\mathbf{R}$. Define $f: X \rightarrow Y$ and $g: X \rightarrow Z$ by

for all $x \in(0,1\rangle f(x)=g(x)=\sin \left(\frac{1}{x}\right)$ and $f(0)=0, g(0)=2$.

It is well known and easy to verify that the function $f$ is Darboux on $X$. But for every $t \in(0,1\rangle$ the set $g(\langle 0, t\rangle)$ is not connected, because it contains the isolated point 2 . Let us remark that $g$ is $f$-continuous on $(0,1\rangle$ but is is not $f$-continuous at the point 0 .

b) Define three topological spaces: $(X, T),(Z, T),(Y, S)$ in this way: $X=Y=$ $Z=\{a, b\}, T=\{\{a, b\},\{a\},\{b\}, \emptyset\}, S=\{\{a, b\}, \emptyset\}$. Define $g: X \rightarrow Z$ and $f: X \rightarrow$ $Y$ by $\forall x \in X g(x)=x$ and $\forall x \in X f(x)=x$. Then the set $f(X)=Y$ is connected in $Y$, but the set $g(X)=Z$ is disconnected, because $Z$ is disconnected too. In this case $g$ is $f$-continuous on $X$, but it is not $f$-constant. Let us observe that the set $X$ is not connected.

Open question. In general, if $g$ is $f$-continuous and $f$ is Darboux, $f$ and $g$ having values in Hausdorff spaces, is $g$ Darboux too?

Evans and Humke defined in [4] the following notion: A function $f: \mathbf{R}^{n} \rightarrow \mathbf{R}$ is Darboux* provided $f$ maps every closed convex set with a nonempty interior to an interval. Our theorem 4.3 says, that if $f: \mathbf{R}^{n} \rightarrow \mathbf{R}$ is Darboux* and $g$ is $f$-constant, then $g$ is Darboux* too. 


\section{References}

[1] Baire, R.: Sur les fonctions des variables réeles. - Ann. Mat. Pura de Appl. 3, 1899, 1-122.

[2] Deimling, K.: Nonlinear functional analysis. - Springer-Verlag, Berlin, Heidelberg, 1985.

[3] Engelking, R.: General topology. - Heldermann Verlag, Berlin, 1989.

[4] Evans, M. J., and P. D. Humke: Revisiting a century-old characterization of Baire class one, Darboux functions. - Amer. Math. Monthly 116:5, 2009, 451-455.

[5] Fuller, R. V.: Relations among continuous and various noncontinuous functions. - Pacific J. Math. 25:3, 1968, 495-509.

[6] Koumoullis, G.: A generalization of functions of the first class. - Topology Appl. 50, 1993, 217-239.

[7] Kupka, I.: On similarity of functions. - Topology Proc. 36, 2010, 173-187.

[8] Kupka, I.: Similar functions and their properties. - Tatra Mt. Math. Publ. 55, 2013, 47-56.

[9] Kuratowski, K.: Topology. Vol. I. - Academic Press, New York, 1966.

[10] LeE, P. Y., W. K. TANG, and D. ZhaO: An equivalent definition of functions of the first Baire class. - Proc. Amer. Math. Soc. 129, 2001, 2273-2275.

[11] O'Malley, R. J.: Baire* 1, Darboux functions. - Proc. Amer. Math. Soc. 60, 1976, 47-56.

[12] Pawlak, H., and R. J. Pawlak: The stable points and the attractors of Darboux functions. - Tatra Mt. Math. Publ. 40, 2008, 13-24.

[13] Pawlak, R. J.: On some class of functions intermediate between the class $B_{1}^{*}$ and the family of continuous functions. - Tatra Mt. Math. Publ. 19, 2010, 135-144.

Received 18 August 2016 • Accepted 12 January 2017 